\title{
Anti-inflammatory and Analgesic Activities of Edgeworthia chrysantha and Its Effective Chemical Constituents
}

\author{
Xiao-Jia Hu, ${ }^{a}$ Hui-Zi Jin, ${ }^{a}$ Wen-Zheng Xu, ${ }^{a}$ Ming Chen, ${ }^{a}$ Xiao-Hua Liv, ${ }^{b}$ Wei Zhang,,${ }^{b}$ Juan Su, \\ Chuan ZHANG, ${ }^{b}$ and Wei-Dong ZHANG ${ }^{*, a, b}$ \\ ${ }^{a}$ School of Pharmacy, Shanghai Jiaotong University; Shanghai 200240, P. R. China: and ${ }^{b}$ Department of Phytochemistry, \\ Second Military Medical University; Shanghai 200433, P. R. China.
}

Received January 22, 2008; accepted May 26, 2008; published online June 23, 2008

\begin{abstract}
The barks and roots of Edgeworthia chrysantha LINDL., which have been used as the folk medicine "Zhu shima" in southern China due to their detumescence and acesodyne effects, were investigated for their antiinflammatory and analgesic activities using a xylene-induced ear edema assay in mice and Freund's complete adjuvant-induced paw edema as inflammation models, and the acetic acid-induced writhing test as an analgesic model. Fractions effective in terms of anti-inflammatory and analgesic activities were obtained from $E$. chrysantha. The chloroform-soluble fraction (CHF) showed significant anti-inflammatory $(p<0.01-0.001)$ and analgesic $(p<0.01)$ effects. On further purification by silica gel, three major coumarins, edgeworin (EdN), edgeworosides $A$ and $\mathrm{C}$ (EdeA and EdeC), were isolated from the chloroform fraction and both anti-inflammatory and analgesic activities were evaluated. EdN and EdeA had anti-inflammatory $(p<0.05-0.01)$ and analgesic $(p<0.001)$ effects, while EdeC only showed an analgesic effect. The results of this study thus demonstrated that the coumarins EdN, EdeA and EdeC in this plant may be active constituents that contribute to the anti-inflammatory and analgesic effects.
\end{abstract}

Key words Edgeworthia chrysantha; anti-inflammatory; analgesic; coumarin

Edgeworthia chrysantha LINDL. is a member of the Thymelaeaceae family. It is distributed in eastern Asia, where the traditional usage of E. chrysantha is very extensive. It is used to make paper in Japan while the flowers are used as the crude drug "Meng hua" which has antibacterial effects. The barks and roots are also used as the folk medicine "Zhu shima" in southern China due to their detumescence and acesodyne effects. The antibacterial properties of this plant have been intensively studied. ${ }^{1,2)}$ However, attention has not been given to other healing properties.

Phytochemical studies revealed that E. chrysantha contains various compounds, such as coumarins, ${ }^{3,4)}$ triterpenes ${ }^{5)}$ and flavonoids, ${ }^{6}$ ) with the former being one of the most common elements in this plant.

Coumarins, identified in various plant families such as $\mathrm{Ru}-$ taceae and Umbelliferae, have extensive anti-inflammatory, analgesic, antifungal, antibacterial and anticruor functions. However, the anti-inflammatory and analgesic effects of the bis-coumarins and coumarin glycosides have not been reported before.

The aim of the present investigation was to evaluate the possible anti-inflammatory and analgesic activities of a $75 \%$ ethanol extract (EEF) of the barks and roots of E. chrysantha and four different fractions partitioned with petroleum ether (PEF), chloroform (CHF), ethyl acetate (EAF) and $n$-butanol (BUF), respectively. Three major constituents from this plant, edgeworin $(\mathrm{EdN})$, edgeworoside A (EdeA), and edgeworoside $\mathrm{C}(\mathrm{EdeC})$, were also tested.

\section{MATERIALS AND METHODS}

Plant Material E. chrysantha used in this study was collected in the flowering season in May 2005 from Nancang, Jiangxi province, P. R. China. A voucher specimen has been deposited at the Department of Phytochemistry of Shanghai Jiaotong University.
Extraction and Isolation of Natural Products The air dried and powdered barks and roots of E. chrysantha $(4.5 \mathrm{~kg})$ were extracted with $75 \%$ ethanol four times $(24 \mathrm{~h} \times 4)$ at room temperature $\left(25^{\circ} \mathrm{C}\right)$. The extract was evaporated under vacuo to afford a residue extract $(500.0 \mathrm{~g})$, which was taken up in water and partitioned in succession with petroleum ether, chloroform, ethyl acetate and $n$-butanol, yielding 102.0 (yield 20.6\%), 78.0 (yield 15.8\%), 115.0 (yield 23.2\%) and $200.0 \mathrm{~g}$ (yield $40.4 \%$ ) of extract, respectively. The CHF fraction $(50.0 \mathrm{~g})$ was subjected to column chromatography on silica gel and eluted with chloroform containing increasing<smiles>O=c1ccc2ccc(Oc3cc4ccc(O)cc4oc3=O)cc2o1</smiles>

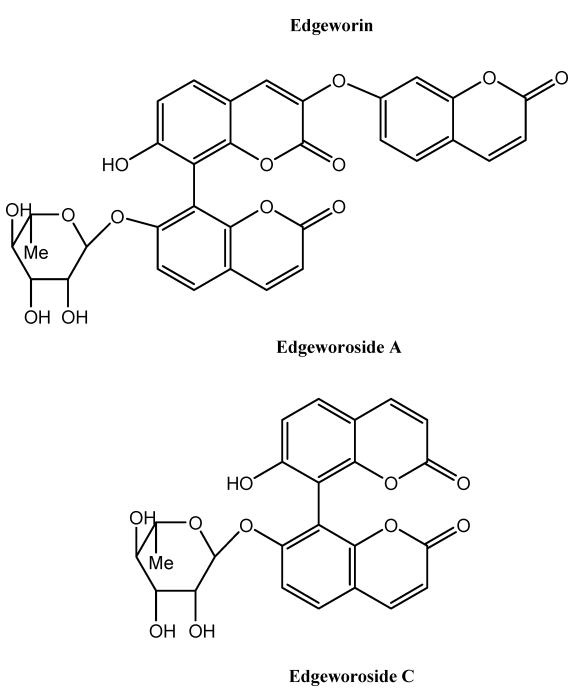

Fig. 1. Structures of Edgeworin (EdN) and Edgeworosides A and C (EdeA and $\mathrm{EdeC})$ 
amounts of methanol. Then EdN (950.0 mg) and EdeA $(1000.0 \mathrm{mg})$ were obtained from a chloroform:methanol fraction $(25: 1)$ and EdeC $(1200.0 \mathrm{mg})$ was isolated from a chloroform: methanol fraction $(5: 1)$. The identity of these main components of $E$. chrysantha was assigned by ${ }^{1} \mathrm{H}-\mathrm{NMR}$ $(400 \mathrm{MHz})$ and ${ }^{13} \mathrm{C}-\mathrm{NMR}(100 \mathrm{MHz})$. The UV, IR, mass, and NMR spectral data of the three compounds were all in agreement with literature values. ${ }^{3,4)}$

Experimental Animals Male ICR mice, weighing 18$20 \mathrm{~g}$ (Experimental Animal Center of Shanghai Pharmaceutical Graduate School, Chinese Academy of Sciences) were used in these studies. All animals were maintained in standard laboratory conditions in the animal care facility (temperature $27 \pm 1^{\circ} \mathrm{C}$ ) under a $12 \mathrm{~h}$ light and dark cycle. They were fed a laboratory diet and had access to water ad libitum.

Male Wistar rats, weighing 200-220 g, (Experimental Animal Center of Shanghai Pharmaceutical Graduate School, Chinese Academy of Sciences) were used in these studies. All animals were maintained in standard laboratory conditions in the animal care facility (temperature $27 \pm 1^{\circ} \mathrm{C}$ ) under a 12-h light and dark cycle. They were divided into groups of five and kept in plastic cages. Food and water were supplied ad libitum.

All animal treatments were performed strictly in accordance with the National Institutes of Health Guide of the Care and Use of Laboratory Animals. The experiments were carried out under the approval of the Committee of Experimental Animal Administration of Second Military Medical University. All experiments were carried out using ten animals per group.

Drugs and Dosage Acetic acid and xylene were purchased from Sinopharm Chemical Reagent Co., Ltd., and both were analytical grade. Aspirin (Asp), hydrocortone (Hyd), dexamethasone (Dex), and indomethacin (Ind) were obtained from Shijiazhuang Pharmaceutical Group Co., Ltd. Aspirin, hydrocortone, indomethacin, 75\% ethanol extract, the four fractions from E. chrysantha, and the EdN, EdeA, and $\mathrm{EdeC}$ were dissolved in purified water with $1 \%$ Tween 80 for both tests. Oral doses of the test fractions and intraperitoneal injection of test compounds were given at a volume of $20 \mathrm{ml} / \mathrm{kg}$ body weight in mice and $15 \mathrm{ml} / \mathrm{kg}$ body weight in rats. Vehicles were used as controls.

Xylene-Induced Ear Edema Assay The test procedure was carried out as described by Hosseinzadeh et al. ${ }^{7)}$ In this experiment, the test fractions from E. chrysantha were administered for five consecutive days and aspirin $(300 \mathrm{mg} / \mathrm{kg}$ ) was given for three days. The pure compounds and Hyd $(25 \mathrm{mg} / \mathrm{kg})$ were administered once intraperitoneally. On the fifth day, $30 \mathrm{~min}$ after p.o. administration of the test fractions and i.p. administration of the test compounds, $0.04 \mathrm{ml}$ of xylene was applied to the anterior and posterior surfaces of the right ear of each mouse. The left ear was considered as a control. One hour after xylene application, the mice were sacrificed and both ears were removed. Circular sections were taken using a cork borer (diameter of $7 \mathrm{~mm}$ ) and weighed. The increase in weight caused by the irritant was measured by subtracting the weight of the untreated left ear section from that of the treated right ear.

Freund's Complete Adjuvant-Induced Paw Edema Assay Adjuvant arthritis was elicited as described by Taurog et $a l .{ }^{8)}$ Briefly, paw volumes of test rats $\left(V_{0}\right)$ were meas- ured at the beginning of the experiment using a plethysmometer (Ugo Basile). The inflammatory response was evaluated by measuring the volume of both paws. The test fractions were administered p.o. for five consecutive days and on the fifth day, $30 \mathrm{~min}$ after oral administration of the test fractions and i.p. administration of the test compounds, the right hind legs were injected with $0.1 \mathrm{ml}$ of Freund's complete adjuvant. The volume $\left(V_{\mathrm{t}}\right)$ of paw edema was measured using a plethysmometer after $18 \mathrm{~h}$ and the increase in volume $\left(\Delta V=V_{\mathrm{t}}-V_{0}\right)$ was recorded. One percent Tween 80 with water was used as control.

Acetic Acid-induced Writhing Test The analgesic effect was tested according to the method described by Shibata et $a l .{ }^{9)}$ Abdomen writhing is a model of visceral pain and was produced by i.p. injection of $0.2 \mathrm{ml}$ of a $0.8 \%$ aqueous solution of acetic acid to each mouse $1 \mathrm{~h}$ after p.o. administration of test extractions and i.p. administration of the test compounds. Immediately after the injection of acetic acid, each was isolated in an individual observation box and the number of abdominal contortions per mouse was counted over a 20 min period.

Cell Culture and Stimulation Murine macrophage RAW264.7 cells were cultured at $37^{\circ} \mathrm{C}$ in Dulbecco's modified Eagle medium (DMEM), containing 10\% fetal bovine serum (FBS), penicillin (100 units/ml), and streptomycin sulfate $(100 \mu \mathrm{g} / \mathrm{ml})$, in a humidified atmosphere containing $5 \%$ $\mathrm{CO}_{2}$. Cells were removed from the tissue culture flask using a cell scraper and resuspended until a final concentration of $1 \times 10^{6}$ cells $/ \mathrm{ml}$. The cells were pretreated with $100 \mu \mathrm{g} / \mathrm{ml}$ of EEF or one of the 4 fractions from $E$. chrysantha $30 \mathrm{~min}$ before being treated with $1 \mu \mathrm{g} / \mathrm{ml}$ lipopolysaccharide (LPS) for $4 \mathrm{~h}$.

Nitrite Oxide (NO) Determination The nitrite concentration in the culture medium was measured as an indicator of NO production based on the Griess reaction by Sherman et $a l .{ }^{10)}$ One hundred microliters of each supernatant was mixed with the same volume of Griess reagent ( $1 \%$ sulfanilamide in 5\% phosphoric acid and $0.1 \%$ naphthylethylenediamine dihydrochloride in water) and the resulting mixture was then incubated at room temperature for $10 \mathrm{~min}$. The absorbance at $540 \mathrm{~nm}$ was then measured using a microplate reader. Freshly cultured medium was used as the blank and LPS as positive control in all experiments.

Acute Toxicity Testing The median lethal doses $\left(\mathrm{LD}_{50}\right)$ of the $75 \%$ ethanol extract of E. chrysantha dried aerial parts (EEF) and four fractions were determined in the mice according to the modified method of Lorke. ${ }^{11)}$ Mice fasted for $24 \mathrm{~h}$ were randomly divided into groups of five mice of either sex per group. Graded doses of the EEF and four fractions were separately administered orally to the mice in each of the test groups. The mice in test groups were then allowed free access to food and water and observed over a period of $7 \mathrm{~d}$ for signs of acute toxicity. The number of deaths (caused by the test drugs) within this period of time were recorded. Logdose response plots are constructed for the plant extract, from which the median lethal doses $\left(\mathrm{LD}_{50}\right)$ of the EEF and four fractions were determined.

Statistical Analysis The results are expressed as the mean \pm S.E.M. The data obtained in experimental groups were evaluated by one-way analysis of variance (ANOVA) followed by Dunnett's $t$-test. Values of $p \leq 0.05$ were consid- 
ered significant.

\section{RESULTS}

The present study was undertaken to investigate the antiinflammatory properties of a $75 \%$ ethanol extract of $E$. chrysantha, four fractions from the extract, and three coumarins $(\mathrm{EdN}, \mathrm{Ede} A$ and $\mathrm{EdeC})$ in response to xylene in mice and Freund's complete adjuvant in rats, and the analgesic effects in response to acetic acid in mice.

Xylene-Induced Ear Edema The results depicted in Fig. 2 show that the administration of EEF $2000 \mathrm{mg} / \mathrm{kg}$ p.o. significantly decreased the xylene-induced mouse ear edema compared with the vehicle treatment group $(p<0.01)$. Among the four subfractions, CHF at $400 \mathrm{mg} / \mathrm{kg}$ p.o. and $\mathrm{EAF}$ at $1200 \mathrm{mg} / \mathrm{kg}$ p.o. also showed significant suppression with inhibition rates of $39.0 \%$ and $30.4 \%$, respectively, while the inhibition rate for Asp $(300 \mathrm{mg} / \mathrm{kg}$, p.o. $)$ was $19.8 \%$. The difference in the inhibition rate between the PEF and BUF groups was not statistically significant.

The three major coumarins obtained from the EEF were also tested and the results are shown in Fig. 3. Significant effects $(p<0.05)$ were observed at high doses $(200 \mathrm{mg} / \mathrm{kg}$, i.p. $)$ of EdN and EdeA, with inhibition rates of $15.1 \%$ and $27.6 \%$, respectively. However, low doses of these two coumarins

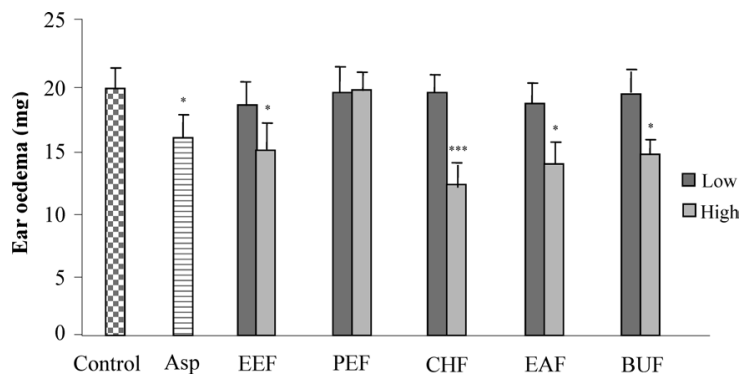

Fig. 2. Effects of the $75 \%$ Ethanol Extract and Four Fractions from $E$. chrysantha on Xylene-Induced Ear Edema in Mice

EEF $(1000,2000 \mathrm{mg} / \mathrm{kg}), \mathrm{PEF}$ and CHF $(200,400 \mathrm{mg} / \mathrm{kg})$, and EAF and BUF $(600$, $1200 \mathrm{mg} / \mathrm{kg}$ ) from E. chrysantha were administered p.o. for five consecutive days. On the fifth day, $30 \mathrm{~min}$ after p.o. administration of the test fractions, $0.04 \mathrm{ml}$ of xylene was applied to the anterior and posterior surfaces of the right ear of each mouse. The left ear served as a control. One hour after xylene application, the mice were sacrificed and the weight of each ear was measured as described in Materials and Methods. Aspirin (Asp $300 \mathrm{mg} / \mathrm{kg}$ ) was used as a positive control. Values are means \pm S.E.M.; $n=10$ mice $* p<0.05$, and $* * * p<0.001$ were compared with the vehicle control group (ANOVA followed by Dunnett's $t$-test).

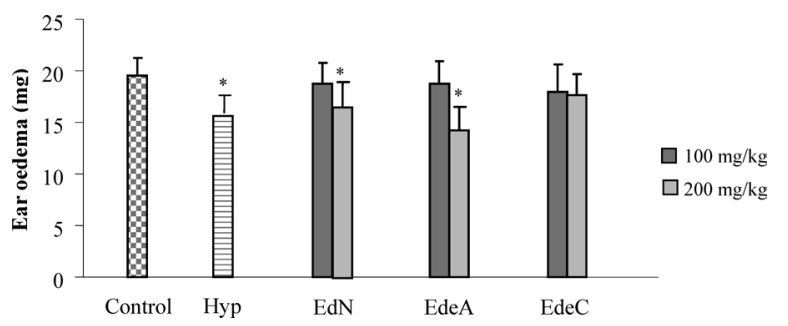

Fig. 3. Effects of Three Coumarins from E. chrysantha on Xylene-Induced Ear Edema in Mice

Doses of EdN, EdeA and EdeC $(100,200 \mathrm{mg} / \mathrm{kg})$ from E. chrysantha were administered i.p. Thirty minutes after administration of the test compounds, $0.04 \mathrm{ml}$ of xylene was applied to the anterior and posterior surfaces of the right ear of each mouse. The left ear served as a control. One hour after xylene application, the mice were sacrificed and the weight of each ear was measured as described in Materials and Methods. Hydrocortone (Hyp, $25 \mathrm{mg} / \mathrm{kg}$ ) was used as a positive control. Values are means \pm S.E.M. $n=10$ mice. $* p<0.05$ was compared with the vehicle control group (ANOVA followed by Dunnett's $t$-test).
$(100 \mathrm{mg} / \mathrm{kg}$, i.p.) and $\mathrm{EdeC}$ at the same dosage had no inhibitory effects. The inhibition rate of Hyp $(25 \mathrm{mg} / \mathrm{kg}$, i.p. $)$ was $20.0 \%$.

Freund's Complete Adjuvant-Induced Paw Edema Orally administered EEF $(1400 \mathrm{mg} / \mathrm{kg})$ showed a significant inhibitory effect $(68.8 \%)$ to Freund's complete adjuvant-induced paw edema in rats $(p<0.01)$. The results are shown in Fig. 4. Inhibitory effects were also observed in the groups treated orally with CHF $(280 \mathrm{mg} / \mathrm{kg})$ and EAF $(840 \mathrm{mg} / \mathrm{kg})$; the percentages of inhibition were $47.1 \%$ and $28.3 \%$, respectively. No significant effects were observed at other doses. The inhibition rate in the positive control group treated with Ind $(210 \mathrm{mg} / \mathrm{kg}$, p.o. $)$ was $35.9 \%$.

It was also observed (Fig. 5) that the edema in rats were inhibited in all of the groups treated with EdN and EdeA, compared to the control group $(p<0.05-0.001)$; the inhibition rates were $56.5 \%(70 \mathrm{mg} / \mathrm{kg}$ i.p. $), 62.5 \%(140 \mathrm{mg} / \mathrm{kg}$ i.p.), $50.0 \%(70 \mathrm{mg} / \mathrm{kg}$ i.p.), and $65.6 \%(140 \mathrm{mg} / \mathrm{kg}$ i.p. $)$, respectively, better than positive control of Dex, which the inhibition was $37.5 \%$, (25 mg/kg i.p.). Meanwhile, EdeC had no effect on the Freund's complete adjuvant-induced paw edema in rats.

Acetic Acid-Induced Writhing In the acetic acid-induced writhing model of analgesic activity, EEF $(2000 \mathrm{mg} / \mathrm{kg}$ p.o.) significantly inhibited the writhing and stretching of

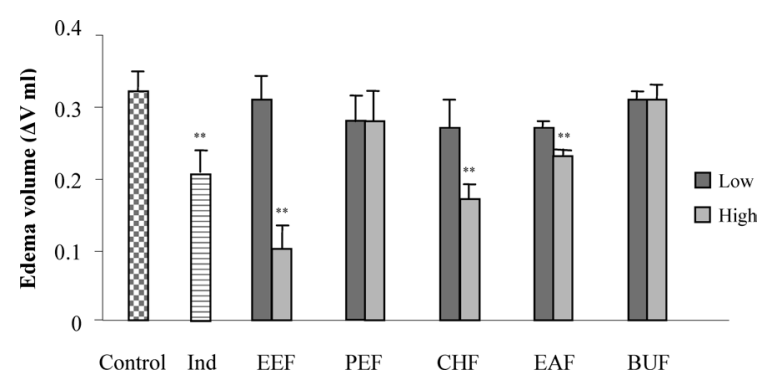

Fig. 4. Effects of the $75 \%$ Ethanol Extract and Four Fractions from $E$. chrysantha on Freund's Complete Adjuvant-Induced Paw Edema in Rats

EEF $(700,1400 \mathrm{mg} / \mathrm{kg}), \mathrm{PEF}$ and CHF $(140,280 \mathrm{mg} / \mathrm{kg})$, and EAF and BUF (420, $840 \mathrm{mg} / \mathrm{kg}$ ) from E. chrysantha were administered p.o. for five consecutive days. Thirty minutes after p.o. administration of the test fractions, the right hind legs were injected with $0.1 \mathrm{ml}$ Freund's complete adjuvant. The volume $\left(V_{\mathrm{t}}\right)$ of paw edema was measured as described in Materials and Methods. Indomethacin (Ind, $210 \mathrm{mg} / \mathrm{kg}$ ) was used as a positive control. Values are means \pm S.E.M.; $n=10$ mice. $* * p<0.01$ was compared with the vehicle control group (ANOVA followed by Dunnett's $t$-test).

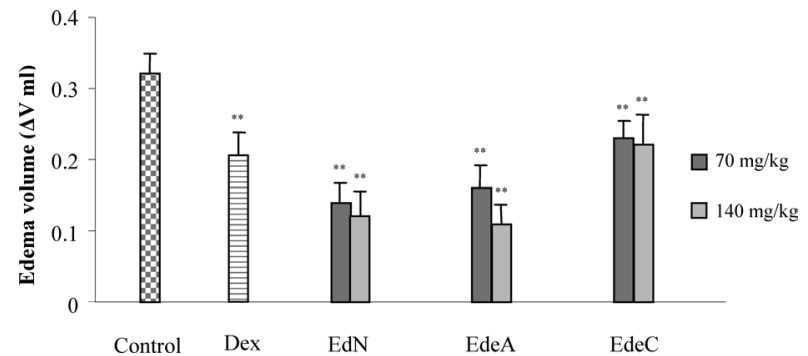

Fig. 5. Effects of Three Coumarins from E. chrysantha on Freund's Complete Adjuvant-Induced Paw Edema in Rats

Doses of EdN, EdeA and EdeC $(70,140 \mathrm{mg} / \mathrm{kg})$ from E. chrysantha were administered i.p. Thirty minutes after i.p. administration of the test compounds, $0.1 \mathrm{ml}$ of Freund's complete adjuvant was injected into the right hind legs. The volume $(V)$ of paw edema was measured as described in Materials and Methods. Dexamethasone (Dex, $25 \mathrm{mg} / \mathrm{kg}$ ) was used as a positive control. Values are means \pm S.E.M.; $n=10$ mice. $* * p<0.01$ was compared with the vehicle control group (ANOVA followed by Dunnett's $t$-test). 


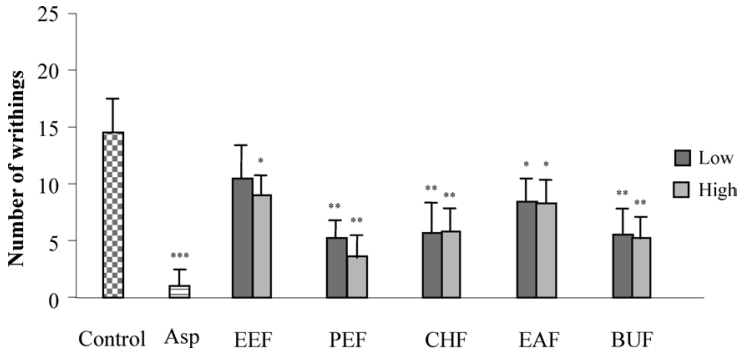

Fig. 6. Effects of the $75 \%$ Ethanol Extract and Four Fractions from $E$. chrysantha on Acetic Acid-Induced Writhing in Mice

EEF (1000, $2000 \mathrm{mg} / \mathrm{kg})$, PEF and CHF (200, $400 \mathrm{mg} / \mathrm{kg})$, and EAF and BUF (600, $1200 \mathrm{mg} / \mathrm{kg}$ ) from E. chrysantha were administered p.o. for five consecutive days. On the fifth day, after i.p. injection of $0.2 \mathrm{ml}$ of $0.8 \%$ aqueous solution of acetic acid to each mouse $1 \mathrm{~h}$ after p.o. administration of test extract, each mouse was isolated in an individual observation box and the number of abdominal contortions per mouse was counted over a $20 \mathrm{~min}$ period. Aspirin (Asp, $300 \mathrm{mg} / \mathrm{kg}$ ) was used as a positive control. Values are means \pm S.E.M.; $n=10$ mice. $* p<0.05$, $* * p<0.01$ and $* * * p<0.001$ were compared with the vehicle control group (ANOVA followed by Dunnett's $t$-test).

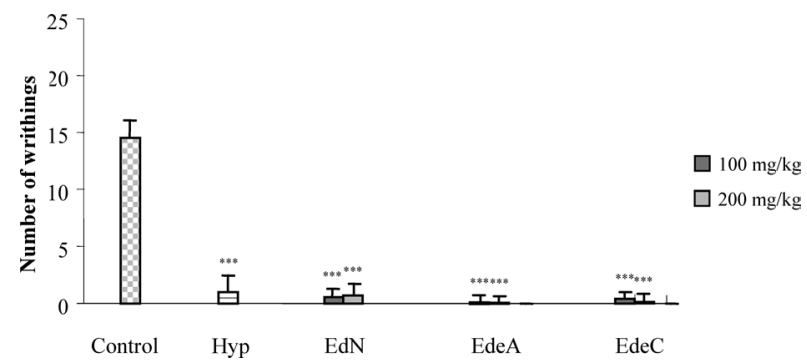

Fig. 7. Effects of Three Coumarins from E. chrysantha on Acetic AcidInduced Writhing in Mice

Doses of EdN, EdeA and EdeC $(100,200 \mathrm{mg} / \mathrm{kg})$ from E. chrysantha were administered i.p. After i.p. injection of $0.2 \mathrm{ml}$ of a $0.8 \%$ aqueous solution of acetic acid to each mouse $1 \mathrm{~h}$ after i.p. administration of the test compound, each mouse was isolated in an individual observation box and the number of abdominal contortions per mouse was counted over a $20 \mathrm{~min}$ period. Hydrocortone (Hyp, $25 \mathrm{mg} / \mathrm{kg}$ ) was used as a positive control. Values are means \pm S.E.M.; $n=10$ mice. $* * * p<0.001$ was compared with the vehicle control group (ANOVA followed by Dunnett's $t$-test).

mice $(p<0.01)$. The groups treated with both doses of PEF, CHF (200, $400 \mathrm{mg} / \mathrm{kg}$ p.o.) and BUF $(600,1200 \mathrm{mg} / \mathrm{kg}$ p.o. $)$ also showed significant effects $(p<0.01)$ (Fig. 6).

The three coumarins were observed to possess significant effects $(p<0.001)$ on acetic acid-induced writhing in mice. The efficacies at both groups of 100 and $200 \mathrm{mg} / \mathrm{kg}$ i.p. of EdeA and EdeC were nearly 10 -fold more potent than positive group of Hyp ( $25 \mathrm{mg} / \mathrm{kg}$, i.p.) (Fig. 7).

NO Test In the NO test, the inhibitory effects of tested samples on NO production were assessed using LPS-induced RAW264.7 macrophages (Fig. 8). The accumulated nitrite, determined by the Griess method, in the medium was used as an index for the NO level. Nitrite content increased markedly with LPS treatment. When the macrophage cells were treated with EEF, CHF, EAF, and BUF at the dose of $100 \mu \mathrm{g} / \mathrm{ml}$, NO production induced by LPS was significantly reduced, however, no reduction in nitrite production was observed in the PEF group. No significant cytotoxicity in the macrophages, as determined by MTT assay, was observed at the concentrations used (data not shown).

Acute Toxicity Testing EEF and four fractions did not exhibit acute toxicity up to the maximum dose of $5 \mathrm{~g} / \mathrm{kg}$ and the weight of the mice was within the normal range after $7 \mathrm{~d}$ of observation. Common side effects such as mild diarrhea, weight loss, and depression were not observed.

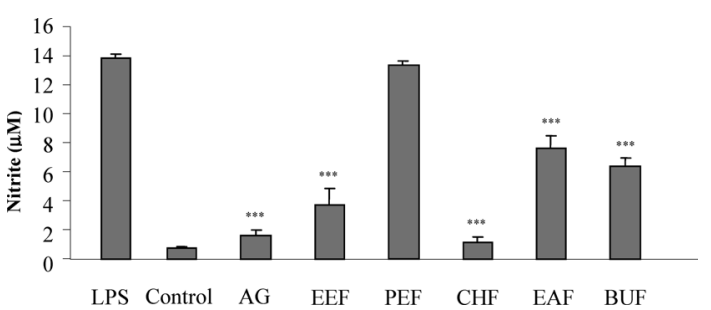

Fig. 8. Inhibitory Effects of EEF and Four Fractions of E. chrysantha on LPS-Induced NO Production in RAW264.7 Macrophage Cells

Murine macrophage RAW264.7 cells were pretreated with EEF $(100 \mu \mathrm{g} / \mathrm{ml})$ or one of the four fractions from E. chrysantha $(100 \mu \mathrm{g} / \mathrm{ml})$ for $30 \mathrm{~min}$, LPS $(1 \mu \mathrm{g} / \mathrm{ml})$ was added, and then the cells were incubated for $24 \mathrm{~h}$. The nitrite concentration in the culture medium was measured as described in Materials and Methods. AG (Aminoguanidine, $3.4 \mu \mathrm{g} / \mathrm{ml}$ ) was used as a positive control. Values are means \pm S.E.M.; $n=10$ mice. *** $p<0.001$ was compared with the vehicle control group (ANOVA followed by Dunnett's $t$-test).

\section{DISCUSSION}

E. chrysantha is a traditional herbal agent which has been used as "Zhu shima" in the treatment and management of anti-inflammatory and painful conditions in China. The flower of this plant is also used in Southern China as an antibacterial agent for the treatment of some eye diseases. However, its pharmacological actions and mechanisms have not yet been precisely documented. This study examined the potential of an ethanol extract of E. chrysantha, four fractions, and three coumarins (EdN, EdeA and EdeC) as anti-inflammatory and analgesic agents in response to xylene, Freund's complete adjuvant, and acetic acid.

In this study, their anti-inflammatory activities were examined using in vivo and in vitro models. In the in vivo models, the inhibition of edema induced by xylene and Freund's complete adjuvant was determined. Xylene-induced mouse ear edema formation is useful for screening and investigating the anti-inflammatory activity of test substances on the acute phase of inflammation. The inhibition rate of the EEF of $E$. chrysantha at an oral dose of $2000 \mathrm{mg} / \mathrm{kg}$ was $25.0 \%$, while that of the positive control Asp (300 mg/kg, p.o.) was $19.8 \%$. Treatment with EEF $(1400 \mathrm{mg} / \mathrm{kg}$, p.o. $)$ decreased the Freund's complete adjuvant-induced paw edema in rats, and the inhibition was nearly twice that of Ind $(210 \mathrm{mg} / \mathrm{kg}$, p.o. $)$. The acetic acid-induced writhing response in mice has long been used as a screening tool for the assessment of analgesic or anti-inflammatory properties of tested samples. ${ }^{12,13)}$ Since EEF was confirmed to have anti-inflammatory effects in xylene-induced edema in mice (Fig. 2) and Freund's complete adjuvant-induced paw edema in rats (Fig. 4), its analgesic effect was examined using an acetic acid-induced writhing model. As shown in Fig. 6, the inhibition rate for EEF at the dose of $2000 \mathrm{mg} / \mathrm{kg}$ p.o. was $37.9 \%$. This result indicates that EEF has a potent analgesic effect in addition to its antiinflammatory effect. Nitrite oxide (NO) is synthesized from L-arginine by nitric oxide synthase (NOS). For the expression of inducible NOS (iNOS), mammalian cells should be triggered by specific stimulants, such as pro-inflammatory cytokines and bacterial LPS. ${ }^{14)}$ Since iNOS-derived NO is involved in various pathological conditions such as inflammation and autoimmune diseases and leads to cellular injury, suppression of iNOS is closely linked with anti-inflammatory action. ${ }^{15)}$ The inhibitory effect of EEF on NO production was assessed by LPS-induced RAW264.7 macrophages (Fig. 8). 
When the macrophage cells were treated with $100 \mu \mathrm{g} / \mathrm{ml}$ EEF, NO production induced by LPS was significantly decreased. The precise mechanism involved in its action is currently not completely understood, but from this finding, it is believed that the anti-inflammatory effect of EEF arises from its inhibition of the release of inflammatory mediators.

In the four fractions of E. chrysantha, CHF displayed both significant anti-inflammatory and analgesic effects $(p<0.01-0.001)$. It is also possible that only a synergistic interaction between the constituents from both CHF and other fractions will demonstrate better anti-inflammatory and analgesic effects. To the best of our knowledge, no systematic studies of the anti-inflammatory and analgesic effects of this plant have been undertaken. The more active components in the CHF and other fractions need to be studied and identified in future research.

Three major coumarins, EdN, EdeA and EdeC, were isolated from $\mathrm{CHF}$ using chromatographic methods. In the acetic acid-induced-writhing model, all three compounds showed potent analgesic effects $(p<0.001)$, while in the antiinflammatory test, the high doses $(200 \mathrm{mg} / \mathrm{kg})$ of $\mathrm{EdN}$ and EdeA showed notable anti-inflammatory effects $(p<0.05$ 0.01 ) and EdeC did not exhibit any significant effect. The anti-inflammatory effects of EdN and EdeA appeared to be dose-dependent.

The reason EdeC displayed analgesic activity only may be related to structural differences between the compounds, and this should be examined in future studies.

\section{CONCLUSION}

In conclusion, the data obtained in this study using xyleneinduced ear edema, Freund's complete adjuvant-induced paw edema, and acetic acid-induced stretching models indicate that the $75 \%$ ethanol extract of E. chrysantha (EEF) possesses significant anti-inflammatory and analgesic activities and that the chloroform fraction (CHF) is the most effective fraction. EEF and CHF reduced NO production stimulated by LPS in RAW264.7 cells. Moreover, three major coumarins, edgeworin (EdN), edgeworoside A (EdeA), and C (EdeC), isolated from CHF were found to be in part respon- sible for the suppressive effects of EEF. More chemical constituents need to be elucidated in future work and further studies are required to determine the possible anti-inflammatory and analgesic mechanisms of actions of these coumarins. Their potential for clinical use also needs to be demonstrated in clinical trials.

Acknowledgments This work was supported by the Program for Changjiang Scholars and Innovative Research Team in University (PCSIRT), NCET Foundation, NSFC (30725045), National 863 Program (2006AA02Z338), Shanghai Leading Academic Discipline Project (B906), and in part by the Scientific Foundation of Shanghai China (07DZ19728, 06DZ19717, 06DZ19005).

\section{REFERENCES}

1) Li X. L., Sun G. J., Dai Sh. P., Li J. S., J. Northwest Pharm., 11, 165166 (1996).

2) Zhang H. J., Zhao Y. Y., Hong S. L., Peng J. R., Chin. Herbal Med., 29, 156-158 (1998).

3) Baba K., Tabata Y., Taniguti M., Kozawa M., Phytochemistry, 28, $221-225$ (1998).

4) Baba K., Taniguti M., Yoneda Y., Kozawa M., Phytochemistry, 29, 247-249 (1990).

5) Ohigashi H., Hirota M., Ohtsuka R., Koshimizu K., Tokuda H., Tennen Yuki Kagobutsu Torokai Koen Yoshishu, 26, 24-31 (1983).

6) Zhang H. J., Zhao Y. Y., Ouyang L., Nat. Prod. Res. Dev., 9, 24-27 (1997).

7) Hosseinzadeh H., Haddadkhodaparast M. H., Arash A. R., Phytother. Res., 17, 422-425 (2003).

8) Taurog J. D., Argentieri D. C., McReynolds R. A., Methods Enzymol., 162, 339-355 (1988).

9) Shibata S., Kumagai A., Harada M., Yano S., Saito H., Takahashi K., U.S. Patent 4374284 (1983).

10) Sherman M. P., Aeberhard E. E., Wong V. Z., Griscavage J. M., Ignarro L. J., Biochem. Biophys. Res. Commun., 191, 1301-1308 (1993).

11) Lorke D., Arch. Toxicol., 54, 275-287 (1983).

12) Tjølsen A., Hole K., Dickenson A., "The Pharmacology of Pain,” Vol. 130, ed. by Besson J., Springer Verlag, Berlin, 1997, pp. 1-20.

13) Franzotti E. M., Santos C. V. F., Rodrigues H. M. S. L., Mourao R. H. V., Andrade M. R., J. Ethnopharmacol., 72, 273-278 (2000).

14) Chesrown S. E., Monnier J., Visner G., Nick H. S., Biochem. Biophys. Res. Commun., 200, 126-134 (1994).

15) Singh V. K., Mehrotra S., Naraya P., Pandey C. M., Agarwal S. S., Immunol. Res., 22, 1-19 (2000). 